\title{
DE LA FILOSOFÍA ANALÍTICA A LA FILOSOFÍA DEL LENGUAJE Y LA HERMENÉUTICA: EL CASO DE LA HISTORIOGRAFÍA
}

From analytical philosophy to philosophy of languaje and hermeneutics: the case of historiography

Mauricio Casanova Brito*

Resumen

Existen dos corrientes de la filosofía analítica en la historiografía: el neopositivismo de Hempel y la filosofía analítica de la historia de Gardiner y Danto. Para el primero, el modelo de la historiografía debe ser similar al de todo conocimiento científico, a saber, el modelo nomotético de las ciencias físicas y naturales. Para Gardiner y Danto, en cambio, este modelo debe desprenderse del propósito particular de los historiadores y del lenguaje acorde a dichos propósitos. Sus conclusiones abrieron la posibilidad de una nueva perspectiva de análisis, el texto historiográfico, lo que impulsó la reflexión hacia la filosofia del lenguaje (White, 1992) y la hermenéutica (Ricoeur, 2008).

Palabras clave: Historiografia, Neopositivismo, Filosofía analítica de la historia, Giro lingüístico, Filosofia hermenéutica.

Abstract

There are two historiographic prospects of analytical philosophy: Hempel's neopositivism and Gardiner and Danto's analytical philosophy of history. According to Hempel, the model of historiography should be the same as all scientific knowledge, namely, the nomothetic model of the physical and natural sciences. According to Gardiner and Danto, however, this model must arise from the purpose of historians and language consistent with those purposes. Gardiner and Danto's conclusions opened the possibility of a new analytical perspective, the historiographic text, prompting the debate toward the philosophy of language (White, 1992) and hermeneutics (Ricoeur, 2008).

Key words: Historiography, Neo-positivism, Analytical philosophy of history, Linguistic turn, Hermeneutic philosophy.

\section{INTRODUCCIÓN}

La filosofía analítica de la historia surge: 1) del rechazo al mentalismo de Collingwood y Croce, al historicismo alemán y a la filosofía sustantiva de la historia, 2) de la superación de la corriente neopositivista de la filosofía analítica anglosajona, y 3) del ímpetu por ofrecer una postura clara de lo que es una explicación historiográfica. Sus postulados principales son: 1) que el mundo al que alude la ciencia positiva y la historiografía es el mismo, 2) por tanto, el carácter metodológico de los estudios históricos no debe desprenderse de las condiciones del objeto de 
estudio, sino 3) del propósito de los historiadores y del lenguaje acorde a dichos propósitos.

Si bien estos postulados nacen de una reflexión epistemológica, heredera de la filosofia analítica anglosajona de principios del siglo XX, las conclusiones que de ellos se desprenden impulsan la reflexión hacia la filosofia del lenguaje y la crítica literaria (White), por un lado, y la hermenéutica (Ricoeur, 2008), por otro, en la medida que: 1) abren la posibilidad de independizar el escrito histórico de la investigación histórica; 2) develan la relación entre la actividad de narrar una historia y el carácter temporal de la existencia humana.

FILOSOFÍA ANALÍTICA DE LA CIENCIA Y FILOSOFÍA ANALÍTICA DE LA HISTORIA: HEMPEL, GARDINER, DANTO

Tanto el neopositivismo de Hempel como la filosofia analítica de la historia tienen su base en la filosofía anglosajona de principios del siglo XX. Ambos consideran que la reflexión en torno al método de una disciplina, ya sea de la historiografía o de la ciencia natural, no debe centrarse en la realidad misma, en el objeto del conocimiento (como en Croce y Collingwood ${ }^{1}$ ), sino en el lenguaje utilizado y su función. Junto con Russell y los positivistas lógicos sostuvieron que el lenguaje formalizado es la matriz lógica del conocimiento científico del mundo y que la labor de la filosofía es reducir este lenguaje a su núcleo lógico (Ankersmit, 11). Sin embargo, las conclusiones que ambas corrientes alcanzaron difieren notoriamente.

Para Hempel, la historiografía debía ajustarse a la estructura lógica de todo lenguaje científico. "Las leyes generales tienen funciones totalmente análogas en la historia y en las ciencias naturales" (307). Es decir: 1) que todas las proposiciones existenciales referentes a entidades o procesos del entorno deben ser validadas por la experiencia; 2) y que dicha referencia debía develarse por el análisis de la estructura lógica de los enunciados. Tal es la idea que Wittgenstein plantea en el Tractatus: que el significado de un enunciado depende de dar un valor de verdad a tal enunciado (Ramírez, 40).

La tarea de los historiadores es dar cuenta de las relaciones causales entre clases de hechos. Con clase Hempel alude a una categoría formal, no a la realidad de la historia. "El objeto de la descripción y explicación en todas las ramas de las ciencias empíricas es siempre la ocurrencia de un hecho de cierta clase" (309). Se refiere no a la revolución soviética o la revolución francesa, sino a la revolución como un tipo de sucesos, similar a una crisis o una guerra. El postulado principal del autor es que:

\footnotetext{
${ }^{1}$ Croce y Collingwood consideran el objeto de la historiografía como una realidad que habita en la mente del historiador (no como una entidad dispuesta en el mundo material). Los hechos y las experiencias del pasado son experiencias mentales y el deber del historiador es revivir imaginariamente lo realizado por los actores del pasado (Croce, 1953; Collingwood, 2004). 
en todos los casos en donde un hecho de una clase $C$ ocurre en un cierto lugar y tiempo, otro hecho de una clase específica $E$ ocurrirá en un lugar y tiempo relacionados de un modo específico con el lugar y tiempo de ocurrencia del primer caso (308).

Las relaciones entre las diversas clases de fenómenos no tienen un compromiso existencial con la realidad, sino que expresan la estructura lógica del lenguaje de la historiografia. En la afirmación de que la revolución francesa fue provocada por la crisis económica que la precedió, está implícito el postulado general, en forma de ley, de que dada una crisis económica es probable que suceda una revolución. Para confirmar esta relación entre las clases crisis económica y revolución, es menester: 1) la formalización clara de las condiciones iniciales y la ley; 2) una investigación empírica exhaustiva. Para Hempel, los historiadores han dejado de lado ambas exigencias, lo que hace de la explicación historiográfica una explicación incompleta. "Lo que los análisis explicativos de los hechos históricos ofrecen es, entonces, en la mayoría de los casos no una explicación [...] sino lo que puede llamarse un esbozo de explicación" (316). La diferencia entre la ciencia natural y la historiografía no es, como se ha pretendido en épocas anteriores, una forma lógica de argumentación, sino simplemente la falta de precisión de las argumentaciones: es una diferencia de grado

Si bien Gardiner comparte con Hempel esta pretensión de evidenciar el método de la historiografía mediante el estudio del lenguaje empleado por los historiadores, no acepta que la estructura lógica del lenguaje de las ciencias naturales constituya el ideal universal de todas las ciencias. Para el autor no existe un lenguaje privilegiado del conocimiento, y la distinción entre las proposiciones utilizadas por las diversas disciplinas obedece principalmente a su función. "El mundo es uno, y diversas las formas que usamos para hablar de él" (Gardiner, 78). La metodología de la historiografía se desprende del examen de "los propósitos de la investigación histórica y [...] los métodos y estructuras conceptuales a estos propósitos" (47).

La premisa principal de La naturaleza de la explicación histórica, obra capital de Gardiner, es que el sentido común (entendido como herramienta para conseguir un fin determinado) es la matriz lógica tanto de la ciencia como de la vida ordinaria. La diferencia entre ambos tipos de lenguaje no son las condiciones de su objeto, sino los fines. "No deseamos ser científicos en todo momento" (36). Para la vida cotidiana es menester que las relaciones entre los fenómenos se perciban de forma inmediata y espontánea. Al contrario de la ciencia, donde el método y la formalidad son primordiales.

La causalidad no es inherente al pensamiento científico, no es una propiedad del mundo al que alude la ciencia, sino que se presenta acorde a su propósito. "La idea de la causalidad es una función de un lenguaje dado, que requiere estar ajustada al nivel particular del lenguaje que se usa" (21). Las causas atribuidas a un determinado efecto no descartan la posibilidad de otras causas dispuestas al mismo 
fenómeno pero en un contexto diferente. "Cuando se hace una afirmación causal de [cualquier] tipo, no se supone que siempre será válida en todas las circunstancias posibles, o que la causa mencionada sea la causa 'verdadera' o 'real' en el sentido de excluir la pertinencia de cualesquiera otras condiciones" (22).

Es un error entonces afirmar que el mundo de la historia es diferente al mundo de las ciencias (idea sostenida por Croce y Collingwood). La diferencia entre la ciencia y la historiografía radica en sus propósitos y no en sus objetos. Cuando un científico escribe sobre un caso particular el lenguaje utilizado forma parte de todo un universo de enunciados y relaciones ya formalizado previamente. Las definiciones de estos conceptos, la mayorías de las veces no mencionados directamente, están ya establecidas. En los estudios históricos no sucede lo mismo. Por este motivo, muchos pensadores han llegado a afirmar que algo anda mal con la historia. Pero ¿por qué debe la historiografía poseer un lenguaje similar al del científico? Gardiner responde:

Hemos visto ya que el historiador no está interesado en formular hipótesis generales o en hacer predicciones; está interesado principalmente en descubrir qué sucedió y en describir lo que sucedió en todos sus detalles; y de esto se desprende que está interesado en hablar acerca de muchos aspectos diversos de la experiencia y la actividad humanas pasadas, a un mismo tiempo. Al historiador, por lo tanto, no le interesa primordialmente el aislamiento de ciertos rasgos de su material, ni la universalización y abstracción extensivas que asociamos con la ciencia (71).

Entonces, la confirmación o refutación de las proposiciones de la historiografía, al contrario de lo que Hempel proponía, no se basa en la adecuación de dichas proposiciones a una ley general:

Una explicación histórica postulada no es, por lo general, justificada (o impugnada) por la demostración de una ley implicada por ella (o no es) válida; mucho menos por la demostración de que tal ley se deriva (o no se deriva) de una teoría o hipótesis aceptada, o de que es confirmada (o refutada) por la experimentación; ni tampoco por la indicación de que el caso en consideración satisface (o no satisface) en los términos requeridos las condiciones exactamente especificadas en la formulación de la ley (116-117).

En lugar de ello, los historiadores insisten en considerar los acontecimientos en todos sus detalles y en toda su complejidad; y mientras más tratamos de apretar las reglas - asevera el autor-, más difícil se torna el juego de la historia.

Danto comparte la mayoría de los postulados de Gardiner. Para el autor de Historia y narración no existen acontecimientos científicos e historiográficos: el mundo es uno, lo que varía es el lenguaje utilizado. El que la historiografía no se acomode a la estructura lógica del lenguaje de las ciencias naturales y físicas no obedece a las particularidades del mundo histórico, sino a que, considerando los propósitos, la información no se puede disponer en el lenguaje formalizado y general de las teorías de la ciencia: 
No existen dos clases de acontecimientos, sino quizá dos clases de descripciones. La ciencia puede ciertamente no conseguir proporcionarnos la información que queremos sobre los acontecimientos, pero eso es porque esa información no siempre se puede formular en el lenguaje abreviado de las teorías científicas" (151).

Para el autor, entre una narración y una crónica no existe un abismo epistemológico: la diferencia no radica en el tipo de conocimiento aplicado. La diferencia es meramente cuantitativa: "no se trata de una diferencia entre clases o géneros de teorías o, para lo que nos ocupa, entre clases y géneros de narraciones: no es sino una diferencia cuantitativa entre grados de confirmación o de fundamentación" (71). No existen, como planteó William Henry Walsh, narraciones puras y narraciones significativas: solo narraciones en diversos grados; y una narración poco documentada y con escaso detalle no constituye una crónica ni una narración exenta de significación: toda narración es significativa.

Cualquier narración está interesada en el hallazgo de la significación de los acontecimientos; idealmente cualquier narración quisiera incluir sólo las cosas relevantes con respecto a otros acontecimientos, o significativas en cuento a ellos. Difícilmente podríamos dividir las narraciones en clases, con este criterio, excepto, quizás, en malas y buenas, siendo las malas las que contienen detalles que no son significativos (Danto, 84).

Para narrar, es menester interpretar. En toda disposición narrativa de los sucesos existe interpretación, siendo esta última no un corolario del texto, un agregado de la descripción, sino el elemento constitutivo de toda narración.

En la medida en que los historiadores describen lo que sucedió por medio de narraciones, se encuentran implicados en algo que se podría denominar "dar una interpretación", puesto que la narración misma es una forma de organizar las cosas y, por ello, "va más allá" de lo dado (95).

La unidad estructural de toda narración son las oraciones narrativas. $\mathrm{Su}$ característica principal es que "se refieren a dos acontecimientos, al menos, separados temporalmente, aunque sólo describen (versan sobre) el primer acontecimiento al que se refieren" (99).

Según Danto, ya en la oración narrativa existe interpretación. Para demostrarlo, ofrece el siguiente caso: imaginemos que existe un mapa completo de los sucesos del pasado, una descripción definitiva, que solamente se extiende en la medida en que el presente deviene en pasado, y que su narrador es un Cronista Ideal:

Sabe todo lo que sucede en el momento en que sucede, incluso en las mentes ajenas. Asimismo tiene el don de la transcripción momentánea: cualquier cosa que sucede a lo largo de todo el borde progresivo del pasado es consignada por él, tal como sucede, en la forma en que 
sucede. Denominaré la relación progresiva resultante Crónica Ideal (de ahora en adelante C.I.) (108).

Considerando que los sucesos pasados están muertos, ya fijados con anterioridad a la narración, solamente con la modificación de estos cambiaría la C.I. Pero como el pasado no sufre modificaciones, la C.I. permanece definitiva. Al contrario, las narraciones de los historiadores están siempre sujetas a posibles cambios dados por la falsedad de los enunciados, por interpretaciones erróneas o por omisiones deliberadas.

Si bien la C.I. puede parecer el destino ideal de toda narración historiográfica, es, para Danto, un regalo envenenado. La C.I. es siempre, aunque parezca una contradicción, incompleta; es completa en el sentido en que un Testigo Ideal puede describir la totalidad de lo que sucede en su entorno, pero no es suficiente.

La verdad completa referente a un acontecimiento sólo puede ser conocida después, y a veces sólo mucho después de que el acontecimiento haya tenido lugar, y sólo los historiadores pueden contar ese relato. Es algo que ni siquiera puede conocer la mejor clase de testigo. Lo que deliberadamente dejamos de proporcionar al Cronista Ideal fue el conocimiento del futuro (112).

La causalidad es solo posible en oraciones narrativas. Aseverar que un suceso A-1 es causa de otro suceso A-2 no es dar cuenta de una unidad ontológica entre ambos acontecimientos, sino solo dar una descripción de A-1 refiriéndose a otro hecho (A-2) que constituye una condición necesaria de A-1 en el contexto de dicha descripción (119-120). De no ocurrir A-1, de ser falso que A-1 tuviera lugar, sería falso también que A-1 causara A-2. No es correcto afirmar que A-1 es una causa suficiente de A-2: antes bien, A-2 es una condición para que A-1 posea el carácter de causa (de A-2); y este tipo de causalidad ocurre solamente en oraciones narrativas, siendo imposibles para la C.I. emplear expresiones como anticipó, dio origen, instigó, causó, provocó, predijo, debido a que para utilizarlas es menester la distancia temporal entre dos sucesos: para aseverar que Lutero inauguró la Reforma, debe haber ocurrido ya la Reforma. El Cronista Ideal solo puede referirse a los hechos presentes; al parecer solo podría decir, usando el ejemplo del autor (121): El 25 de diciembre de 1642 nació Isaac Newton. Sin embargo, para nosotros, dicha frase es también una oración narrativa si consideramos el significado que tiene Isaac Newton en el mundo posterior a 1642 y pasado para nosotros. Para la C.I. todos los hechos son igual de significativos $\mathrm{y}$, por ende, igual de insignificantes. En una oración narrativa, en cambio:

una cosa u ocurrencia particular adquiere significación histórica en virtud de sus relaciones con alguna otra cosa u ocurrencia, en la que resulta que tenemos especial interés, o a la que damos importancia por la razón que sea. Así pues, las oraciones narrativas que usan frecuentemente para justificar la mención, en una 
narración, de alguna cosa o acontecimiento, cuya significación se le escaparía de otro modo a un lector (135).

En síntesis, tanto Gardiner como Danto, ambos herederos de la filosofía analítica anglosajona, consideraron: 1) que el mundo de la ciencia y la historiografía es el mismo; 2) que el método de la historiografía obedece a sus propósitos (para Gardiner, describir lo que sucedió en todos sus detalles, para Danto, comprender el significado del proceso estudiado) y; 3) que el lenguaje utilizado por los historiadores (para Gardiner, la asociación causal particular, para Danto, la narración) debe dar cuenta de dichos propósitos.

\section{DE LA FILOSOFÍA ANALÍTICA AL GIRO LINGÜÍSTICO-HERMENÉUTICO: WHITE Y RICOEUR}

La obra de Gardiner y Danto es esencialmente una reflexión epistemológica. Surge: 1) del rechazo al mentalismo de Collingwood, al historicismo alemán y a la filosofía sustantiva de la historia; 2) del ímpetu por ofrecer una postura clara de lo que es una explicación historiográfica. En este punto no difieren de los análisis realizados por Walsh o Hempel. Sin embargo, la atención exhaustiva al lenguaje de los historiadores impulsó el debate hacia otro terreno, ajeno hasta ese entonces, para la teoría de la historiografia: el texto.

Así, en las décadas de 1950 y 1960, la filosofia de la historia prefirió dedicarse a los elementos del texto histórico, como declaraciones singulares acerca de situaciones pasadas, declaraciones que expresan conexiones causales, o a la perspectiva temporal de las declaraciones sobre el pasado (los «enunciados narrativos» de Danto). El texto histórico en conjunto rara vez, si acaso alguna, fue objeto de investigación filosófica (Ankersmit, 15).

Gardiner y Danto proporcionaron a la filosofía del lenguaje, todavía heredera del atomismo lógico de Russell, la posibilidad de un campo nuevo de estudio caracterizado por la autonomía de la investigación (cuyas conclusiones se manifiestan en declaraciones particulares sobre el pasado) por sobre el texto (que incorpora las consecuencias culturales de la investigación al conjunto de la escritura). Sin embargo, la posibilidad de esta nueva perspectiva no significó un tránsito inmediato desde la filosofia de la historia epistemológica hacia la filosofía de la historia lingüística. Al contrario, sin desmerecer los aportes de Gallie, Louch y Dray, el giro a la lingüística de la historiografía se anuncia solamente con la publicación de Metahistoria de White en 1973.

En Metahistoria White busca analizar más allá de los datos, conceptos teóricos o estructura narrativa de la obra histórica - lo que se puede considerar como la superficie o el nivel manifiesto-. En cambio, propone el entendimiento de la historiografía como un acto poético, es decir, el acto en el cual el historiador "prefigura el campo histórico y lo constituye como un dominio sobre el cual aplicar las teorías específicas para explicar lo que 'en realidad estaba sucediendo' en él” (10). 
White intenta así hacer explícitos y visibles, por medio de posibilidades de prefiguración tropológica, las conceptualizaciones previas con las cuales el historiador concibe la realidad del pasado. Tanto la historia como la filosofía de la historia, dirá el autor, combinan datos y conceptos con el fin de representar un conjunto de acontecimientos que supuestamente ocurrieron en tiempos pasados. Sin embargo, ni una se agota en el uso de datos y su agrupación en tipologías generales que engloben hechos y procesos. Estas también poseen un contenido estructural profundo e inconsciente de naturaleza poética. El contenido funciona como paradigma lingüístico metahistórico presente en todas las obras de historiografia.

Para el autor, en la operación en que una crónica —entendida como un conjunto de acontecimientos dispuestos en orden cronológico- se convierte en un relato poseedor de comienzo, desarrollo y fin, existen modalidades que provienen de la precomprensión del historiador del entorno histórico-social en el que está inmerso. Estas modalidades son las que, en última instancia, le otorgan sentido a la narración historiográfica: son elementos de traducciones del pasado, similar a los componentes léxicos, gramatical, sintáctica y semántica de un texto escrito. White los clasifica en tres grupos: 1) modo de tramar (la explicación de un relato mediante el reconocimiento del tipo de relato), 2) argumentación formal (la explicación de lo sucedido aludiendo a principios de combinación que funcionan como leyes), 3) implicación ideológica (el elemento ético en la posición del historiador en torno a la naturaleza del conocimiento histórico y sus implicaciones en el presente).

Este procedimiento de traducción está siempre guiado por uno de los cuatro tropos: metáfora, sinécdoque, metonimia e ironía. La teoría de los tropos es la base para clasificar las estructuras profundas de imaginación histórica tanto de la historiografía como de la filosofía de la historia. Ofrece recursos útiles para "comprender las operaciones por las cuales los contenidos de experiencia que se resisten a la descripción en prosa clara y racional pueden ser captados en forma prefigurativa y preparados para la aprehensión consciente" (43). Para White, en toda traducción del pasado la operación de asociar un fenómeno con otro supone la existencia de un contenido estructural profundo e inconsciente de naturaleza poética: este contenido no se describe por medio de ninguno de los fenómenos de la asociación (por ejemplo, en la metáfora mi amor una rosa, los fenómenos amor y rosa). Tampoco el mecanismo de asociación obedece a la lógica o a lazos de carácter epistemológico: al contrario, su criterio es estético y no referencial (Iggers, 35).

El acto de interpretación, que en Danto no excedía la esfera de la comprensión historiográfica, en White se extiende a todo el pasado:

Igual que un texto, el pasado posee un significado que tratamos de descubrir, necesita interpretación, y consiste en elementos léxicos, gramaticales, sintácticos y semánticos. Por tanto, lo que en esencia hace el historiador es traducir el texto del pasado al narrativo del historiador (Ankersmit, 129-130). 
Tomando distancia del debate en torno al estatuto epistemológico de la historiografía, White permite comprender el texto histórico como el resultado de un acto de composición estética similar a los de la filosofía y la literatura. La filosofía epistemológica de la historiografía de Gardiner y Danto se transforma ahora en una filosofía del lenguaje histórico; no solo del lenguaje de la historiografía, sino de toda interpretación del pasado, ya sea científica, artística o historiográfica.

Con Ricoeur esta pregunta por las condiciones para entender el sentido de un texto o de la misma historia, presente en toda Metahistoria, se reemplaza por la pregunta por las condiciones de todo comprender. La pregunta por los niveles particulares del comprender se transforma en la pregunta por aquel ser que es en la medida que comprende. La reflexión deviene desde el comprender como modo de conocimiento hacia el comprender como modo de ser:

La pregunta: ¿cuáles son las condiciones necesarias para que un sujeto cognoscente pueda comprender un texto, o la misma historia?, se sustituye por esta otra pregunta: ¿qué es un ser cuyo ser consiste en comprender? El problema hermenéutico se convierte así en una región de la Analítica de ese ser, el Dasein, que existen al comprender (El conflicto, 11).

Pero esta sustitución no es inmediata. Al contrario, es por la vía larga del estudio del universo de las significaciones particulares ya objetivadas, más que por la vía corta de la ontología del ser finito, que Ricoeur procura develar el carácter originario de toda comprensión. Pretendiendo no empantanarse en una filosofía del lenguaje a la manera de Wittgenstein o en una filosofia reflexiva de tipo neokantiana (12), el autor enfatiza no tanto en los elementos preformativos o formales del lenguaje, como en White, sino en la manera en que en dicho lenguaje opera el tiempo. Para ello, es menester el estudio exhaustivo de dicho lenguaje: "¿acaso no es nuevamente en el lenguaje mismo donde debe buscarse la pauta de que la comprensión es un modo de ser?" (15).

Para Ricoeur, el texto tiene siempre un sentido múltiple que va más allá de la organización interna de la obra. En el universo simbólico del texto, en donde "un sentido directo, primario y literal designa por añadidura otro sentido indirecto, secundario y figurado, que sólo puede ser aprehendido a través del primero" (17), se dice más de lo que se dice, y nunca se termina de dar que decir (32). El texto, y todo el lenguaje, lo concibe el autor no como un espacio simbólico cerrado y autosuficiente de signos, sino como un universo abierto que obedece al juego del devenir entre lo heredado y lo interpretado.

Desde este punto de vista, el de la temporalidad, el texto se ubica en una zona intermedia entre lo circunstancial y lo sistémico: entre el tiempo de transmisión y el tiempo de interpretación; entre experiencia vivida y lenguaje. Es una manera de vivir el tiempo — caracterizada por el advenimiento del sentido- que permite la sedimentación del depósito de la tradición y la explicitación de la interpretación: que hace posible la lucha entre la temporalidad que transmite y la que renueva (31). El 
texto permite articular aquello que fue historia y devino mundo objetivado (estructura/sistema inconsciente constituido por diferencias y oposiciones independientes al observador) con aquel acto de continuación consciente de un fondo simbólico llevado a cabo por un intérprete ubicado en el mismo campo semántico donde se ubica la comprendido, ingresando así en el círculo hermenéutico (55).

Con la carga temporal del texto Ricoeur pretende expresar que la afinidad con la tradición es originaria y constitutiva de la finitud del estar ahí tanto como el hecho de que este estar ahí se proyecte siempre a sus posibilidades futuras: que las condiciones de estar arrojado y proyectado no deben pensarse separadamente.

Esta vía larga de la hermenéutica de Ricoeur toma como objeto a la historiografía en el primer tomo de Tiempo y narración. La idea principal es que:

entre la actividad de narrar una historia y el carácter temporal de la existencia humana existe una correlación que no es puramente accidental, sino que se presenta como forma de necesidad transcultural. Con otras palabras: el tiempo se hace tiempo humano en la medida en que se articula en modo narrativo, y la narración alcanza su plena significación cuando se convierte en una condición de existencia cultural (113).

Lo central de la identidad estructural de la narración no es ni el estatuto epistemológico de la historiografía (Danto) ni la prefiguración poética tras la construcción de una trama (White), sino el carácter temporal de la existencia humana. "El mundo desplegado por toda obra narrativa es siempre un mundo temporal" (39).

Para Ricoeur, esta mediación entre tiempo y narración se devela en "el papel mediador de la construcción de la trama entre el estadio de la experiencia práctica que la precede y que la sucede" (115). Al estadio de la experiencia práctica lo denomina el autor, utilizando el concepto aristotélico de mímesis (imitación creativa de la acción): mímesis I. Al de la construcción de la trama (texto historiográfico): mímesis II. Al campo de la refiguración de nuestra experiencia: mímesis III. El círculo hermenéutico entre las tres mímesis representa el paso entre un tiempo prefigurado (mímesis I) a otro refigurado (mímesis III) por medio de uno configurado (mímesis II).

En primer lugar, toda composición de una trama encuentra siempre sus raíces en la precomprensión del mundo de la acción (mímesis I). "Si es cierto que la trama es una imitación de la acción, se requiere una competencia previa: la de identificar la acción en general" (116). El autor considera aquí tres aspectos: 1) aspectos estructurales: una acción adquiere significado en su relación con los demás componentes estructurales de una red conceptual. El concebir estos componentes constituye la comprensión práctica del hacer. La acción implica fines (el qué de la acción o lo que se hace en ella), motivos (el porqué de la acción), agentes (el quién de la acción), circunstancias (el cómo de la acción) y resultados; 2) aspectos simbólicos del campo práctico: el símbolo se ubica siempre en un campo de significación público, que es siempre independiente y ya heredado al agente que realiza y comprende la acción; 3) aspectos temporales: el agente de la acción es un agente 
atento, es decir, su presente permanece en la atención que se dilata en la espera que se va convirtiendo en memoria.

La mímesis II, que corresponde al plano de la configuración del tiempo, es la mediadora entre la precomprensión del mundo de la acción (mímesis I) y la operación de lectura y amplificación de la realidad (mímesis III). Aquí, la construcción de la trama es mediadora en tres aspectos: 1) en primer lugar, entre acontecimientos singulares y una historia concebida como un todo. En la construcción de una trama el historiador le otorga un lugar a la infinidad de hechos individuales dispersos en el campo histórico. Estos acontecimientos pierden así su carácter de accidental o ser disperso. De la contribución del hecho a la historia como un todo se desprende el significado de este; 2) en segundo lugar, la construcción de la trama integra los elementos dispersos en la precomprensión estructural de la acción (fines, motivos, agentes, circunstancias y resultados esperados e inesperados) y les otorga un lugar único dentro de una historia con comienzo, desarrollo y fin. Al complejo universo de motivos aparentemente azarosos y universales el historiador les quita su carácter accidental; 3) por último, la trama es mediadora entre una dimensión cronológica y otra no cronológica. La primera hace alusión a la sucesión episódica de acontecimientos. La segunda es la operación que configura, que transforma este devenir episódico en una historia concebida como totalidad.

Por último, la mímesis III constituye el campo de la intersección entre el mundo del escritor, el mundo del texto y el mundo del oyente o lector. Aquí, el acto de la lectura es el punto mediador entre mímesis II y mímesis III, mientras que la operación de ampliación de la realidad es el nexo entre mímesis III y mímesis I.

Lo que recibe el lector no es solo el sentido de la obra, sino también por medio de este, el universo de significado del mundo y la temporalidad. Pero no nos referimos a una transmisión exacta, en el sentido de un agente que ofrece un contenido ya configurado a otro agente que tiene solamente el rol de recibir. Tanto el lector como el texto participan de la imaginación creadora: ambos son partes de la composición de la trama. "La construcción de la trama solo puede describirse como un acto del juicio y de la imaginación creadora en cuanto que este acto es obra conjunta del texto y de su lector" (147). El acto de la lectura es siempre un procedimiento de actualización del texto. El sentido de la obra no se agota en la estructura interna del texto (tampoco en su prefiguración poética, como en White), antes bien, el sentido se obtiene de la fusión entre texto y la referencia del interlocutor. Esta referencia es siempre correferencia o referencia dialogal.

El texto historiográfico se sustrae así del horizonte vital del autor. Toda exégesis permanece viva en este círculo de significación que se desvincula de la pura psicología del autor. "Al liberarse la escritura, no solo de su autor sino también de la estrechez de la situación dialogada, revela su destino de discurso como proyección de un mundo" (Villarroel, 64). 
En síntesis, el acto de interpretación, que en Danto no excedía la esfera de la comprensión historiográfica, en White se extiende a todo el pasado, develando así el carácter poético y preformativo de todo texto histórico. Luego, con Ricoeur, en el universo simbólico de este último se manifestará no solamente su naturaleza poética sino la equivocidad misma (posibilidad de entenderse o interpretarse en varios sentidos) del ser. Como Heidegger declaró en Ser y tiempo, no es posible pensar de forma aislada la condición de estar arrojado en el mundo, por un lado, y la disposición a la posibilidad, por otro: ambas condiciones se manifiestan ya en el comprender originario que constituye el ser del ser ahí.

\title{
CONCLUSIONES
}

Dos son las conclusiones principales de la obra de Gardiner y Danto. Con respecto al primero: la afirmación de que el carácter metodológico de la historiografía se desprende no tanto del examen de las particularidades del objeto de estudio (del pasado), sino de la finalidad de los historiadores (el estudio de procesos históricos particulares) y del lenguaje acorde a dicha finalidad. Con respecto al segundo: la afirmación del carácter incompleto de toda narración; la narración histórica en Danto no es un mero vehículo de transmisión de información, es un procedimiento de continua producción de significado.

Estas conclusiones abren la posibilidad de: 1) independizar el escrito histórico de la investigación histórica, y; 2) dar cuenta de la relación entre la actividad de narrar una historia y el carácter temporal de la existencia humana. Esta primera posibilidad se manifiesta en Metahistoria. La segunda, en Tiempo y narración.

Con White la filosofía epistemológica de la historiografia de Gardiner y Danto se transforma en una filosofía del lenguaje histórico; no solamente del lenguaje de la historiografía, sino de toda interpretación del pasado, ya sea científica, artística o historiográfica. Los procedimientos correctos de una explicación historiográfica pierden importancia: los aspectos centrales son ahora los elementos prefigurativos y poéticos de toda interpretación del pasado.

Luego, con Ricoeur, la reflexión excederá el estudio de estos elementos prefigurativos: el acto de configuración de una trama se entenderá ahora como una operación mediadora entre la precomprensión del mundo de la acción y el acto de lectura y amplificación de la realidad. En este círculo hermenéutico, en donde el intérprete se ubica en el mismo campo semántico donde se ubica lo comprendido, el tiempo (en un primer instante, de naturaleza aporética) devendrá en tiempo humano. Para la hermenéutica de Ricoeur, la narración no es una mera forma de explicación propia de la historiografía, como en Danto, sino una condición de existencia cultural

\author{
Universidad de Concepción* \\ Serrano 62, Santiago (Chile) \\ mauriciocasanova@gmail.com
}




\section{OBRAS CITADAS}

Ankersmit, Frank. Historia y tropología. México: Fondo de Cultura Económica, 2004.

Collingwood, Robin George. Idea de historia. México: Fondo de Cultura Económica, 2004.

Croce, Benedetto. Teoría e historia de la historiografia. Buenos Aires: Imán, 1953.

Danto, Arthur. Historia y narración: ensayos de filosofia analítica de la historia, Barcelona: Paidós, 1989.

Gardiner, Patrick. La naturaleza de la explicación histórica, México: Universidad Nacional Autónoma de México, 1961.

Hempel, Carl. La explicación científica. Estudios sobre filosofia de la ciencia. Barcelona: Paidós, 2005.

Iggers, Georg. La historiografia del siglo XX. Desde la objetividad cientifica al desafio posmoderno. Chile: Fondo de Cultura Económica, 2012.

Ramírez, Alejandro. La transformación de la epistemología contemporánea: de la unidad a la dispersión. Santiago: Editorial Universitaria, 2005.

Ricoeur, Paul. El conflicto de las interpretaciones. Ensayos de hermenéutica. Buenos Aires: Fondo de Cultura Económica, 2008. Tiempo y Narración I. Buenos Aires: Siglo XXI, 1995.

Villarroel, Raúl. "Ética y medio ambiente. Ensayo de hermenéutica referida al entorno", Revista de Filosofia, 63 (2007): 55-72.

Walsh, William Henry. Introducción a la filosofia de la historia, México: Siglo XXI Editores, 1970.

White, Hayden. Metahistoria, la imaginación histórica en la Europa del siglo XIX. México: Fondo de Cultura Económica, 1992. 